\title{
VERTICAL MICROLENS SCANNER FOR 3D IMAGING
}

\author{
Sunghoon Kwon, Veljko Milanovic, ${ }^{\dagger}$ and Luke P. Lee \\ Berkeley Sensor and Actuator Center \\ Department of Bioengineering, University of California, Berkeley, CA 94720 \\ ${ }^{\dagger}$ Adriatic Research Institute, Berkeley, CA 94704-1029
}

\begin{abstract}
We have designed, fabricated, and demonstrated a static vertical microlens scanner with large static displacement and low $(<10 \mathrm{~V})$ driving voltage, using silicon-on-insulator (SOI) technology. The unique isolated vertical combdrive sets and the coupled-torsion flexure design provides for both upward and downward piston motions and the low driving voltage. Singledirectional devices demonstrate maximum static downward displacement of $8 \mu \mathrm{m}$ at $10 \mathrm{Vdc}$ actuation voltage. Bi-directional devices demonstrate vertical actuation from $-6.5 \mu \mathrm{m}$ to $+9 \mu \mathrm{m}$ also at sub-10 $\mathrm{V}$. The devices have mechanical resonant frequencies near $400 \mathrm{~Hz}$, and when operated at resonance, a vertical displacement of up to $55 \mu \mathrm{m}$ peak-to-peak is achieved at up to 7 $V_{r m s}$. The lens motion shows near pure piston motion with very small tilt angle of less than $0.034^{\circ}$ and the compensation of the tilt using an isolated comb bank is demonstrated.
\end{abstract}

\section{INTRODUCTION}

Three dimensional (3D) raster scanning of focused light is a basic function for many photonic applications because one can focus light on arbitrary points located in 3D space. A miniaturized $3 \mathrm{D}$ raster scanning module with low power and low cost can enable very challenging and exciting optical MEMS applications such as high throughput 3D imaging with array of MEMS microscopes [1], retinal displays [2], free space optical communication networks with micro motes [3], and 3D MEMS memory [4]. Advancement of miniaturized optical components provided by optical MEMS is a very promising solution to implement miniaturized, cost effective 3D raster scanning module.

We have been developing miniaturized 3D raster scanning module, which can be fit into $1 \mathrm{~mm}^{3}$ as a key part of micro confocal imaging array for lab-on-a-chip applications. The diagram for transmissive scanning using three microlens scanner is shown in Fig. 1. High-speed 3D raster scanning of focus can be implemented in shortest optical path only normal to substrate by stacking independent transmissive microlens scanners. While light is passing through each scanner moving in orthogonal direction at different speed, light is steered as well as focused. Successful 2D raster scanning at a focal plan has been demonstrated by stacking of two lateral combdrive actuated microlens [1].

However, vertical actuation of microlens is necessary to complete 3D raster scanning by varying effective focal length of whole system. Pure out of plane piston motion of a microlens can control beam divergence, and set the focal plane to desired level without moving sample. Furthermore, importance of vertical microlens actuator is clear when we consider the light emitting and sensing direction of a solid state light source and a detector such as VCSEL and photodiode, and desired hybrid integration between

Contact email address: lplee@socrates.berkeley.edu

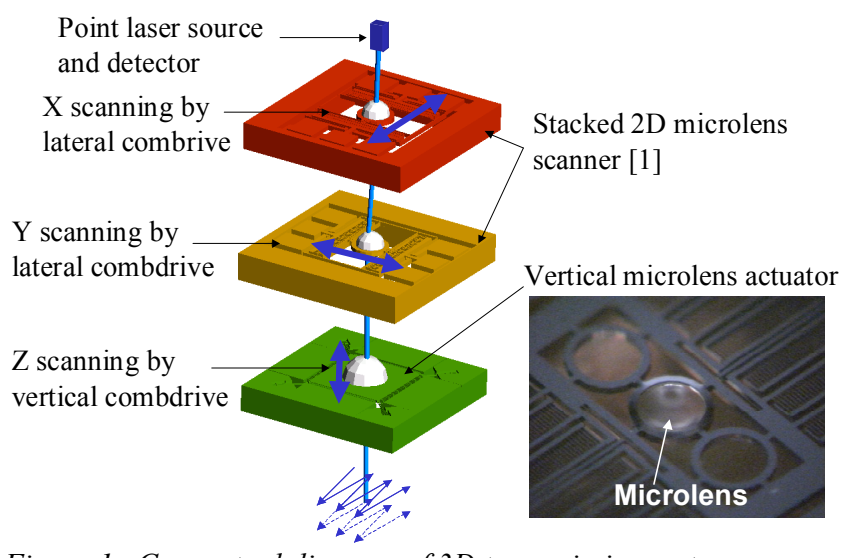

Figure 1. Conceptual diagram of $3 D$ transmissive raster scanner.

silicon MEMS based microoptical components and III/V compound based solid state light sources. Vertical combdrive actuator with limited displacement has been reported using combined bulk and surface micromachining [5]. Because the device layer thickness determines the maximum displacement of vertical comb drive, thick device layer such as SOI is favorable to achieve large vertical displacement. However, the trade-off between large displacement and driving voltage limits the performance of such devices due to the high vertical spring constant in high aspect ratio structure.

In this paper, we present the design, fabrication and characterization of SOI vertical actuators with integrated microlenses for 3D imaging application. The features such as large vertical displacement with sub-10 volt actuation via isolated vertical comb banks and a symmetric coupled torsion flexure, and tilting correction capability to pure vertical motion are described with a key enabling multi-level beam SOI-MEMS fabrication technology.

\section{DESIGN CONSIDERATIONS}

The desired vertical actuation of microlens for hand-held 3D imaging should meet following important requirements: linear force generation in overall dynamic range for controllability, low power consumption, large static and dynamic vertical displacement for large scanning range, pure piston motion to minimize aberration from lens tilting, and compensation capability for possible lens tilting to minimize optical aberrations. Electrostatic vertical comb drive can meet the first two requirements if it has pre-engagement, [5] i.e. some fabricated overlap of upper and lower comb fingers. Vertical comb actuator for our specification should have such pre-engagement for constant force over the range of motion and high aspect ratio for large displacement and optical flatness. Because our 3D imaging application demands at

This work is funded by DARPA BioFlips program. Travel support has been generously provided by the Transducers Research Foundation and by the DARPA MEMS and DARPA BioFlips programs. 


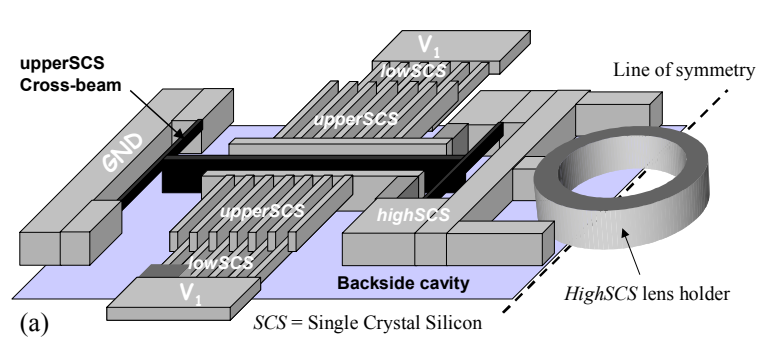

Coupled torsion flexure

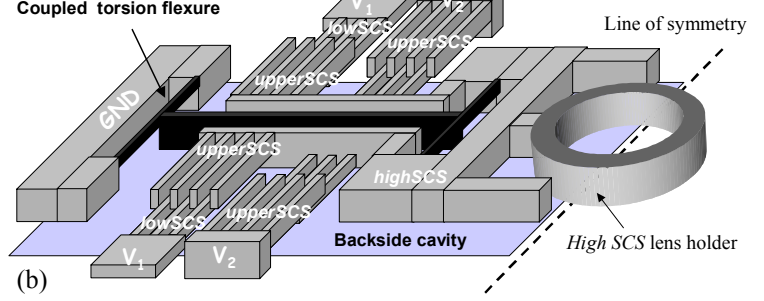

Figure 2. Schematic of one side of the high aspect ratio vertical actuator based on isolated vertical combdrives for : (a) unidirectional, and (b) bi-directional piston motion

minimum $10 \mu \mathrm{m}$ of static displacement range and comb finger preengagement for best control, previously demonstrated vertical comb drives [2],[5] do not satisfy both requirements. Furthermore, independently controllable comb banks via isolation are favorable to compensate possible lens tilting due to the mismatch from fabrication, and also for the first time to allow actuation both up and down, thereby doubling the total static scanning range. The isolated high aspect ratio vertical comb drive with pre-engagement can be ideal solution for these requirements.

The schematic of one side of the high aspect ratio vertical actuator based on isolated vertical combdrives for unidirectional and bi-directional piston motion is shown in Fig. 2. The devices consist of three major units: isolated comb banks, a symmetric coupled torsion flexure, and a thick lens frame. This is an exact analogy to lateral comb drive actuators so that one can design a vertical combdrive exactly same way as a lateral combdrive. The electrostatic force generated by vertically defined comb drives pull the thick lens holder downward or upward. The two symmetric coupled torsional flexures on both sides of the lens holder transform the torsion of the beam to pure piston motion of the lens.

The isolated comb bank is defined over three different design layers which denote single-crystal-silicon (SCS) beams etched at different vertical levels: highSCS, lowSCS and upperSCS [6] as depicted in Fig. 3(a). The highSCS layer, the full thickness SOI device-layer beam, is used for rigid structures such as lens holder and connecting beams. The lower comb fingers are defined in the lowSCS layer. And compliant torsional flexures and upper comb fingers are defined in the upperSCS. LowSCS and upperSCS layer can have arbitrary thickness due to timed etching. We chose the goal for thickness of both layers to be $3 / 5$ of full device layer thickness, such that there is $1 / 5$ thickness of overlap (for comb finger pre-engagement,) as shown in Fig. 3a. Because all these three different layers are in SOI device layer, the isolation between comb banks is achieved, which leads to independent actuation between comb fingers for bi-directional motion and tilting compensation. In the Fig. 2(b), the schematics shows four isolated combdrive sets with control voltages $\mathrm{V}_{1}, \mathrm{~V}_{2}$. Opposite end of device is controlled by $\mathrm{V}_{3}, \mathrm{~V}_{4}$. Normal upward motion is powered by $\mathrm{V}_{2}, \mathrm{~V}_{4}$ while downward motion is powered by $\mathrm{V}_{1}, \mathrm{~V}_{3}$. Tip/tilt compensation is possible with independent control of $V_{1}-V_{4}$. Applying additional compensation DC voltage to the other side of line of symmetry can compensate the tip/tilt in the line of symmetry axis.

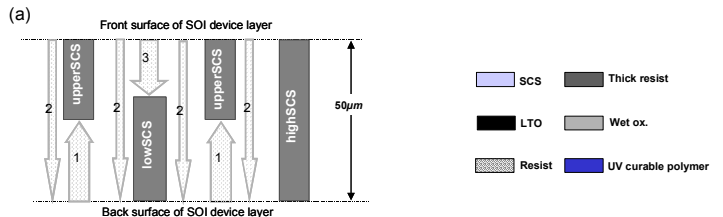

(b)
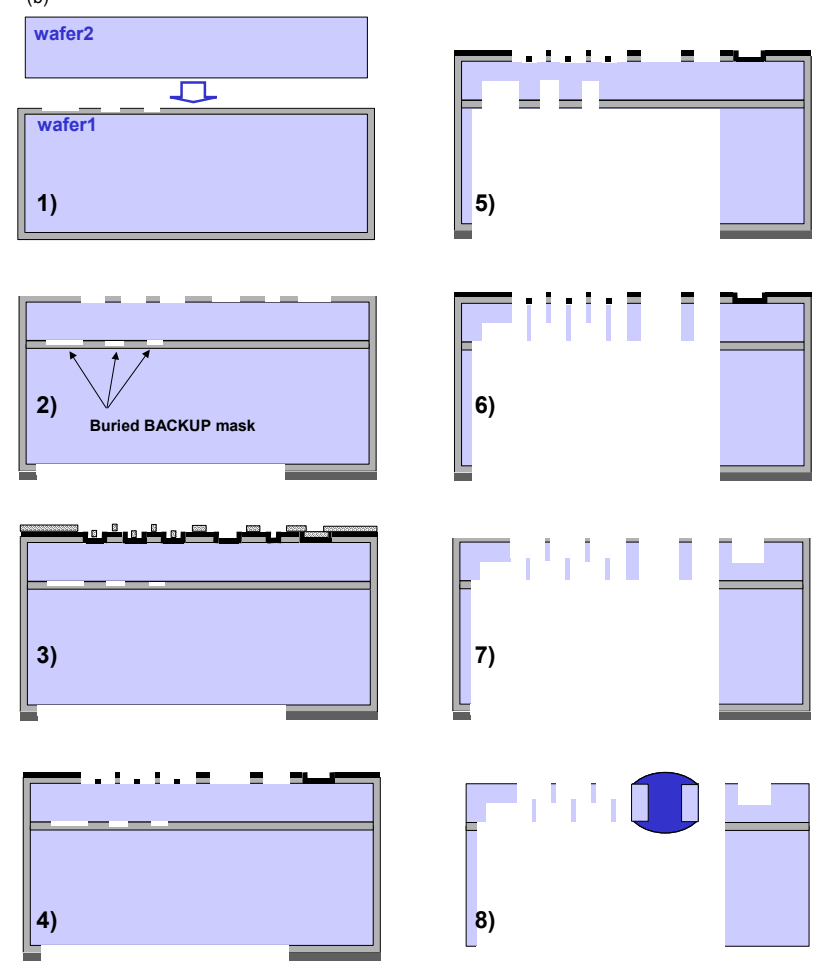

Figure 3. Fabrication process: (a) Conceptual diagram showing order of etching, and (b) detailed fabrication step for masking layer(1-4), etching (5-7), and lens fabrication (8).

The symmetric coupled torsion flexures enables low voltage performance by substituting the difficult out-of-plane bending of high aspect ratio thick beams with more compliant torsion. If we compare the compliance of our coupled torsion flexure with conventional double folded flexures, the coupled torsion flexure can be more than 80 times compliant than the double folded with the same length. The compliance ratio between the conventional bending type and this coupled flexure is a strong function of aspect ratio. As aspect ratio increases the proposed coupled torsion flexure becomes more compliant than the bending type, which makes it attractive in high aspect ratio SOI MEMS. This unique design provides minimum lateral instability and tilting of the lens holder while allowing low-voltage piston motion. And finally the ring shape lens holder is defined in the thick highSCS layer for integration of microlenses. The inner and outer diameters of ring shape lens holder are design parameters related with required numerical aperture and entrance pupil size.

\section{FABRICATION}

The fabrication process for a vertical microlens scanner is outlined in Fig. 3. The three design layers explained in the previous section are conceptually depicted in Fig. 3(a). In addition to these three layers, the backside of the device was opened to allow an optical window for the microlens, large piston motion, and dry releasing of the device. Such a multilevel beam SOIMEMS process has been demonstrated for laterally driven torsional micromirror applications [6]. In that process the minimum possible pattern size for the Backup mask layer (which 


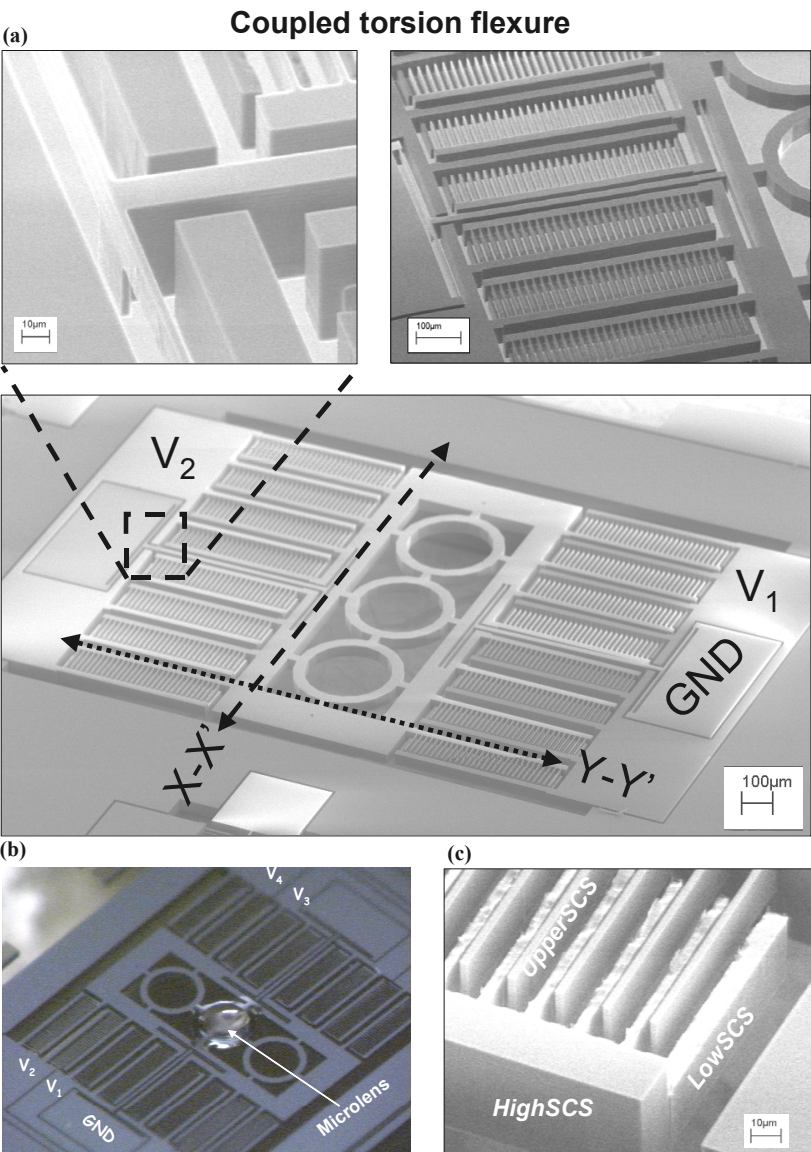

Figure 4. SEM and picture for fabricated vertical microlens scanner: (a) SEM of unidirectional device, (b) Microscopic picture of bi-directional device with polymer lens, (c) self aligned vertical comb fingers.

undercuts device SOI from the backside to create UpperSCS beams) was limited to tens of $\mu \mathrm{m}$ because the mask was applied to the SOI wafer backside and mapped to the SOI isolation oxide by a long DRIE. Therefore, it was impossible to place upper and lower beams (here used as upper and lower comb-fingers) within $\sim 20$ $\mu \mathrm{m}$. The advanced version of that process, utilized in this work, performs pre-etching of the backup mask in buried isolation oxide layer during SOI wafer bonding, enables more accurate definition of upperSCS features as well as minimum feature distance between various beam types. Namely, in the advanced process, all the critical, beam-defining masks are prepared from the topside using the wafer-stepper for accurate alignment. Another advancement over the previous process is the mask self-alignment, which is critical capability for useful vertical comb-drive based devices. We utilize a single photoresist mask to align both the upperSCS and LowerSCS etch-defining oxide masks, as shown in Fig. 3(b).

The devices were fabricated in the Berkeley Microlab using Adriatic Research Institute multilevel beam SOI-MEMS technology. The detailed fabrication steps are as follows: 1) $2 \mu \mathrm{m}$ of thermal oxide is deposited to the first double polished wafer and backup mask is patterned into the oxide layer. Another wafer is thermally bonded and grinded to desired device layer thickness, 50 $\mu \mathrm{m}$. 2) $1.4 \mu \mathrm{m}$ of thermal oxide is deposited and the first front side mask for highSCS and backside mask for defining optical window and releasing device. This frontside mask is actually bigger than the desired highSCS to allow alignment margin to next layer. 3) $600 \mathrm{~nm}$ of LTO is deposited and the mask for lowSCS and highSCS is patterned to resist layer, which means both lower and upper comb fingers are self aligned in same mask [7], which prevents the critical alignment step. All pattern size is desired size in this step. 4) LTO and Thermal oxide is etched by same selfalign resist mask from 3). Now all masks making step is done. 5) Large backside window for optical window is opened by DRIE, followed by the $20 \mu \mathrm{m}$ thinning of upperSCS layer from the backside using buried backup mask. 6) HighSCS and lowSCS are etched from the front also by DRIE, followed by remaining thin LTO timed etching by RIE. 7) LowSCS is thinned by the front side DRIE. The design rule includes the possible lateral etching of lowSCS. 8) UV curable polymer droplet is applied to highSCS ring shape lens holder and surface tension induces perfect lens shape as previously characterized in [1]. Exposure to ultra violet solidifies the polymer droplet on $300 \mu \mathrm{m}$ lens holder in diameter. The fabricated unidirectional and bi-directional devices are shown in Fig. 4(a) and (b). The X-X' line in the figure matches with the line of symmetry in Fig. 2. The self-aligned vertical comb fingers, which $0.1 \mu \mathrm{m}$ accurate alignments is accomplished easily using the self-align method, are shown in Fig. 4(c).

\section{RESULTS AND DISCUSSION}

Static and dynamic characteristic of the vertical microlens actuator is measured using a laser vibrometer. Figure 5(a) shows the downward static vertical displacement for both unidirectional and bi-directional design. Figure 5(b) shows the upward vertical motion for bi-directional device. The devices have large vertical displacement upto $\pm 10 \mu \mathrm{m}$ at sub-10V driving voltage. This low voltage actuation is owing to compliant coupled torsion flexure as well as high density vertical comb banks enabled by buried design of the coupled flexure, which allows more area for comb drives. The maximum displacement at $388 \mathrm{~Hz}$ in resonance was above 50 $\mu \mathrm{m}$ and the scanning range control using linear amplitude modulation is possible as shown in Fig. 5(c). The frequency
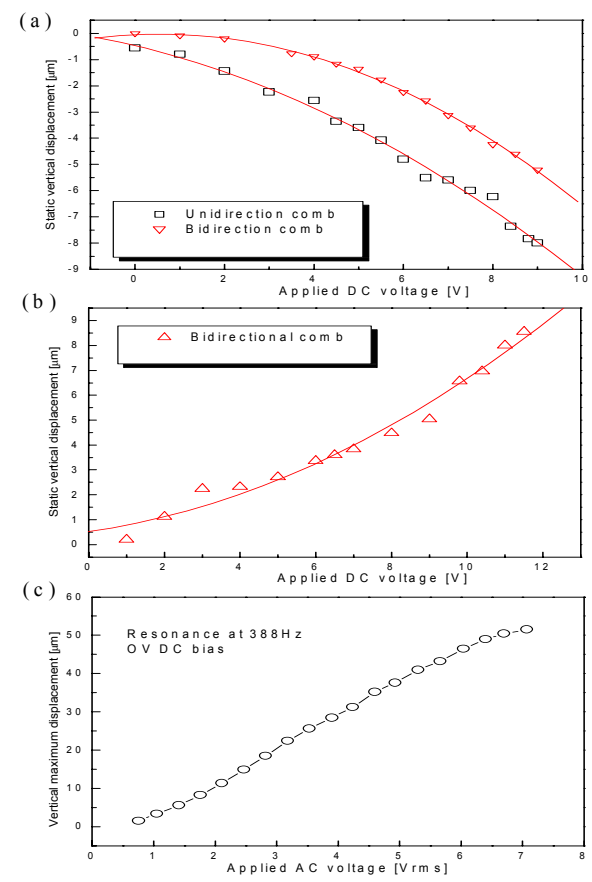

Figure 5. Static and dynamic characterization of the devices: (a) static DOWNWARD vertical deflection of both the singledirectional and the bi-directional device, (b) UPWARD vertical deflection of the bi-directional device, and (c) peak-to-peak dynamic deflection at $388 \mathrm{~Hz}$. 

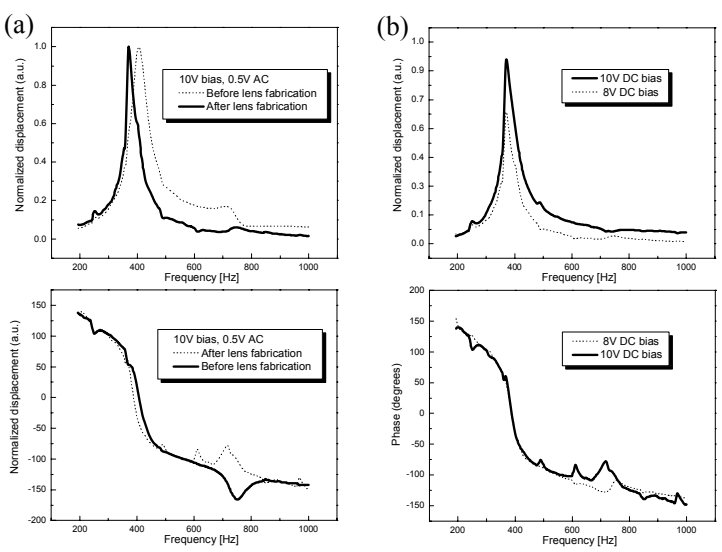

Figure 6. Frequency response of vertical microlens actuator : (a) before and after lens fabrication, and (b) DC bias effect on resonance frequency.

responses for before and after lens fabrication along with DC vias effect are measured using a laser vibrometer and a lock-in amplifier as shown in Fig. 6. Resonance frequency decreased by only $10 \%$ by increased mass from lens. Also the DC bias effect on resonance frequency was less than $5 \%$ if we compare the frequency response for $10 \mathrm{~V}$ and $8 \mathrm{~V}$ DC bias.

The tilting of lens plate during the actuation is measured for estimating possible degree of optical aberration using interferometric profiler. The measured tilt of $\mathrm{X}-\mathrm{X}^{\prime}$ axis and $\mathrm{Y}-\mathrm{Y}^{\prime}$ axis denoted in Fig. 7(a) is shown in Fig. 7(a). Basically the device has maximum tilting of $0.034^{\circ}$ during piston motion in $\mathrm{Y}^{-} \mathrm{Y}^{\prime}$ axis, and $\mathrm{X}-\mathrm{X}^{\prime}$ axis has one tenth of tilting compared with $\mathrm{Y}-\mathrm{Y}^{\prime}$ 'axis. This small tilting angle is negligible for most of applications, however, the compensation to zero tilt is also possible by using
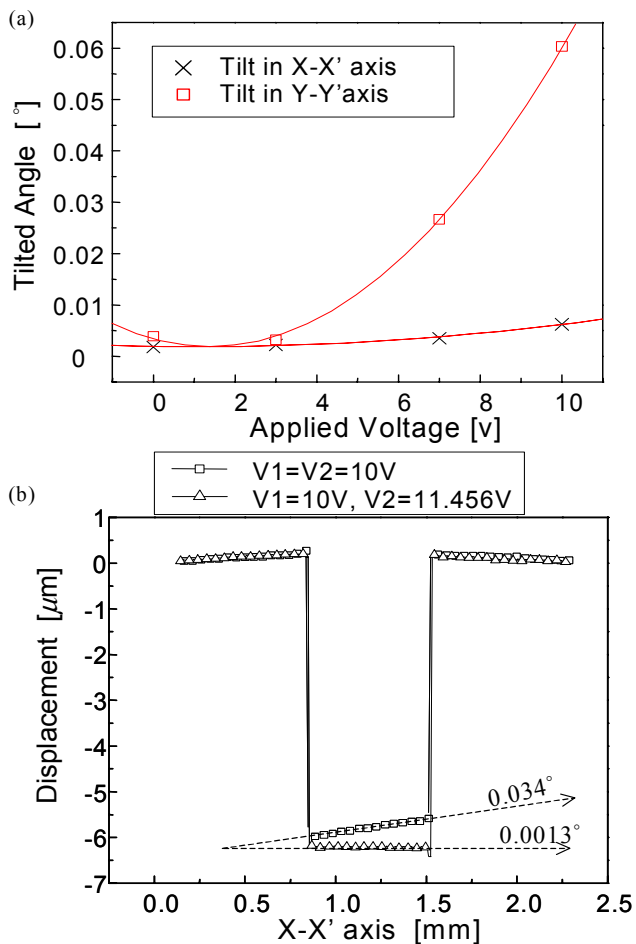

Figure 7. Tilt compensation experiment result showing: (a) Tilt of lens plate for symmetric driving voltage, and (b) tilt compensation by asysmetric driving voltage. Tilt reduced from $0.034^{\circ}$ to $0.0013^{\circ}$ after compensation. asymmetric driving using featured isolated comb banks. To demonstrate the compensation capability using our isolated comb bank, which is one of the major advantage of our process, we tried to compensate the $\mathrm{X}-\mathrm{X}^{\prime}$ axis tilting by applying asymmetric voltage to either side of line of symmetric. In other word, by applying asymmetric voltage to $\mathrm{V}_{1}$, and $\mathrm{V}_{2}$ indicated in Fig. 4(a). The compensation result is shown in Fig. 7(b) by acquiring profile along with Y-Y'axis. The rectangle profile is symmetric actuation with $10 \mathrm{~V}$ and the tilt of lens frame was $0.034^{\circ}$. The tilt is compensated to $0.0013^{\circ}$ using asymmetric actuation voltages of 10 $\mathrm{V}$ and $11.456 \mathrm{~V}$ in either side of isolated comb banks, which are the electrodes V1 and V2, respectively. Theoretically, the perfect tilting compensation is possible using this technique.

\section{CONCLUSIONS}

We have demonstrated low voltage and large static displacement vertical actuators with integrated microlenses on high aspect ratio silicon-on-insulator (SOI). Due to the coupled torsion flexure and isolated vertical comb banks, low voltage pure vertical piston motion of microlens is demonstrated. The developed device is not only key part for our continueing effort for micro confocal microscope array but also attractive to hybrid integration of photonic devices.

\section{REFERENCES}

1. S. Kwon, and L. Lee, "Stacked Two Dimensional Microlens Scanner for Micro Confocal Imaging Array", 15th Annual IEEE International MEMS 2002 Conference, 1/20-24/02, Las Vegas (2002), pp 483-486.

2. R. Conant, J. Nee, K. Lau, and R. Muller, "A Flat HighFrequency Scanning Micromirror," ", Technical Digest of the 2000 Solid-State Sensor and Actuator Workshop, Hilton Head Isl., SC, 6/4- 6/8, Transducer Research Foundation, Cleveland (2000)

3. B. Warneke, M. Last, K. Liebowits, and K.S.J. Pister, "Smart Dust: Communicating with a Cubic-Millimeter Computer", Compueter Magazine, January 2001, pp.44-51.

4. Y. Kawata, "Three-dimensional Memory", Proceedings of the SPIE - The International Society for Optical Engineering, vol.4081, SPIE-Int. Soc. Opt. Eng, 2000. p.76-85.

5. Yeh, J.-L. A., Hui, C.-Y., and Tien, N. C., "Electrostatic Model for an Asymmetric Vertical Combdrive", Journal of Microelectromechanical systems, Vol. 9, No. 1, Mar. 2000.

6. V. Milanovic, M. Last, and K.J.S. Pister, "Torsional Micromirrors with Lateral Actuators", Transducers'01 Erosensors XV conference, Muenchen, Germany, Jun. 2001.

7. U. Krishnamoorthy, O. Solgaard, "Self-Aligned Vertical Combdrive Actuators for Optical Scanning Micromirrors," Optical MEMS'01, Okinawa, Japan, Sep. 2001. 